\title{
Facile Synthesis and Characterization of Zinc Tetranitro Phthalocyanine-MWCNTs Nanocomposites with Efficient Visible-Light-Driven Photocatalytic Activity
}

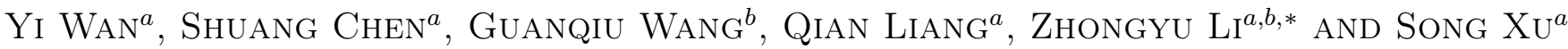 \\ ${ }^{a}$ Jiangsu Key Laboratory of Advanced Catalytic Materials and Technology, School of Petrochemical Engineering, \\ Changzhou University, Changzhou 213164, PR China \\ ${ }^{b}$ College of Hua Loogeng, Changzhou University, Changzhou 213164, PR China
}

(Received February 6, 2016; in final form June 17, 2016)

\begin{abstract}
Novel zinc tetranitro-phthalocyanine (ZnTNPc) supported by multi-walled carbon nanotubes hybrid composites were facilely prepared by a method of ultrasonic impregnation and their photocatalysis behavior was studied. The as-prepared ZnTNPc-MWCNTs composites were characterized by scanning electron microscopy, Xray diffraction, diffuse reflectance spectra, transmission electron microscopy, thermogravimetric analysis, and the Fourier transform infrared spectra. The results showed that the ZnTNPc was not only grown on the multi-walled carbon nanotubes but also uniformly disturbed without aggregation. Compared with pure ZnTNPc and MWCNTs, ZnTNPc-MWCNTs nanocomposites presented a significantly enhanced photocatalytic activity for the degradation of rhodamine B under visible-light irradiation. Furthermore, a possible mechanism for the photodegradation of rhodamine B was also proposed.
\end{abstract}

DOI: 10.12693/APhysPolA.130.785

PACS/topics: 78.67.Bf; 81.05.Zx; 81.07.Pr

\section{Introduction}

Photocatalysis for the degradation of organic pollutants has gained increasing attention as a promising technique [1-4]. A large amount of photocatalytic materials have been investigated, including $\mathrm{TiO}_{2}[5-7], \mathrm{ZnO}[8,9]$, $\mathrm{CdS}[10,11], \mathrm{g}-\mathrm{C}_{3} \mathrm{~N}_{4}[12,13]$, etc. Among the reported photocatalytic materials, multi-walled carbon nanotubes (MWCNTs) as a novel metal free photocatalyst support have good photocatalytic performance under visible-light irradiation [14, 15], and it also demonstrates the property of low cost, non-toxicity, and thermal stability [16]. Therefore, MWCNTs have been widely used in the degradation of organic pollutants and the direct production of clean hydrogen. On the other hand, since the chemical bonding of MWCNTs is solely via $s p^{2}$ bonds similar to those of graphite, the high-level electron mobility and electrical conductivity of MWCNTs allow them to use as supports for macrocyclic organic catalysts such as metalloprophyrins and metallophthalocyanines (MPcs). Hence, there is a need to develop an efficient visible light responsive photocatalyst to extend its spectral response to higher absorption wavelength.

Phthalocyanines, especially those metal phthalocyanines, could be referred to as attractive alternatives for the visible-light-included photocatalytic decomposition of dye compounds [17-21]. Photoactivity of phthalocyanines arises from their ability to produce the high active

\footnotetext{
*corresponding author; e-mail: zhongyuli@mail.tsinghua.edu.cn
}

${ }^{1} \mathrm{O}_{2}$ singlet oxygen species upon photonflux absorption in either UV or visible parts of the spectrum. This singlet oxygen is formed during the extinction mechanism of the excited triplet state, following its collision with molecular $\mathrm{O}_{2}$. In addition, besides this property, metallophthalocyanines revealed extraordinary molecular stability, negligible toxicity and high thermal stability. The nanoscale particles could enhance the photocatalytic activity. Zinc phthalocyanine ( $\mathrm{ZnPc})$ and its derivatives possess a wide visible light response $(600-800 \mathrm{~nm})[22$ 24]. They can be synthesized with different substituent groups and combined with other compounds by loading or coordination. The synthetic flexibility of phthalocyanines offers great possibilities to modify the length of the connection groups and the positions of substituted groups [25]. Furthermore, zinc phthalocyanine is able to photochemically activate triplet oxygen into singlet oxygen $\left({ }^{1} \mathrm{O}_{2}\right)$, which is frequently used as a non-radical oxidant for oxidizing organic pollutants [26]. However, metallophthalocyanines are easy to aggregate, leading to markedly decrease the catalytic activity. Therefore, combining the photoresponsive property of both MWCNTs and ZnTNPc to prepare a photocatalyst with favorable dispersibility and higher photocatalytic activity seems to be extraordinarily vital.

In the present study, the ZnTNPc-MWCNTs hybrid materials were prepared by an ultrasonic impregnation method. The photocatalytic performance of asprepared ZnTNPc-MWCNTs nanocomposites was evaluated by the degradation of rhodamine $\mathrm{B}(\mathrm{RB})$ under visible-light irradiation $(>420 \mathrm{~nm})$. It was found that the ZnTNPc-MWCNTs photocatalysts could significantly enhance photocatalytic activity. Furthermore, a 
possible mechanism for enhancing photocatalytic activity of ZnTNPc-MWCNTs was also discussed.

\section{Experimental}

\subsection{Synthesis of $Z n T N P c$}

All chemicals were reagent grade and used without further purification. In a typical preparation procedure, $3.828 \mathrm{~g}$ (4 mmol) 3-nitrophthalonitrile, $1.835 \mathrm{~g}$ (1 mmol) zinc acetate, 1 -pentanol $(100 \mathrm{ml})$ and DBU $(1.5 \mathrm{ml})$ were put into a three-necked $100 \mathrm{ml}$ round bottom flask. The mixture was then stirred at $130^{\circ} \mathrm{C}$ for $6 \mathrm{~h}$. After reaction the reaction mixture was diluted with methanol and the precipitate was filtered off. Finally, the purified ZnTNPc (its structure formula is shown in Fig. 1) was obtained after washing by $1 \mathrm{~mol} / \mathrm{L} \mathrm{NaOH}$ and $1 \mathrm{~mol} / \mathrm{L} \mathrm{HCl}$.

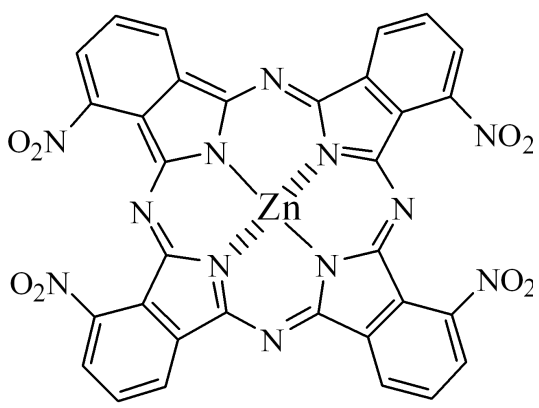

Fig. 1. Structural formula of ZnTNPc.

\subsection{Preparation of ZnTNPc-MWCNTs hybrid materials}

The ZnTNPc-MWCNTs hybrid materials were prepared via a method of ultrasonic impregnation. Before preparing the ZnTNPc-MWCNTs hybrid materials, multi-walled carbon nanotubes must be purified in order to remove amorphous carbon fibers, amorphous carbon particles and other impurities such as graphite particles. In brief, $2 \mathrm{~g}$ of MWCNTs was grinded in the agate mortar for $20 \mathrm{~min}$, and then added to $100 \mathrm{ml}$ of concentrated nitric acid, sonicated for $30 \mathrm{~min}$ to obtain a well dispersed MWCNTs solution. The suspension was magnetically stirred and regurgitated at $120^{\circ} \mathrm{C}$ for $10 \mathrm{~h}$, then cooled down to room temperature naturally, washed with absolute ethanol and distilled water repeatedly, then dried under vacuum at $60^{\circ} \mathrm{C}$ for $12 \mathrm{~h}$ to obtain the carboxylic acid-functionalized MWCNTs. The synthesis procedure of ZnTNPc-MWCNTs is as follows: $0.4 \mathrm{~g}$ ZnTNPc were added into $100 \mathrm{ml}$ ethanol under magnetic stirring. Then $0.1 \mathrm{~g}$ oxidized-MWCNTs were added and sonicated for $2 \mathrm{~h}$. The mixture solution was then centrifuged and the supernatant was collected. Finally, the ZnTNPcMWCNTs were obtained after dried in a vacuum at $80^{\circ} \mathrm{C}$ for $4 \mathrm{~h}$.

\subsection{Characterization}

XRD analyses were carried out with a Rigaku D/Max2500PC X-ray diffractometer (Rigaku Co.,Japan), using a graphite crystal monochromator to select the $\mathrm{Cu} K_{\alpha 1}$ radiation source at $\lambda=1.5406 \AA$, with a step size of $0.02 \mathrm{~s}^{-1}$. Morphologies of the prepared samples were observed on a JSM-6360LA scanning electron microscope (SEM, JEOL, Japan) and a JEM-2100 transmission electron microscope (TEM, JEOL, Japan). UV-vis diffuse reflectance spectra (DRS) of the samples were recorded by a UV-vis spectrophotometer (Shimadzu UV-2550). Fourier transform infrared spectra (FT-IR) of samples were collected with a Nicolet (Protégé 460) spectrometer in the range from 500 to $4000 \mathrm{~cm}^{-1}$. The thermal stability of the materials was carried out using the TG-209F3 thermogravimetric analysis meter (Nestal Company, Germany).

\subsection{Photocatalytic test}

The photoreactor was designed with an internal xenon lamp (XHA $1000 \mathrm{~W}$ and the average intensity was $28 \mathrm{~mW} / \mathrm{cm}^{2}$ ) equipped with a cut-off glass filter transmitting $>400 \mathrm{~nm}$ surrounded by a water-cooling quartz jacket to cool the lamp, where a $100 \mathrm{~mL}$ aliquot of the $\mathrm{RB}$ solution with an initial concentration of $25 \mathrm{mg} / \mathrm{L}$ in the presence of ZnTNPc-MWCNTs hybrid catalysts (50 mg). The volume of initial RB solution is $50 \mathrm{ml}$. The solution was stirred in the dark for 30 min to obtain a good dispersion and established adsorption-desorption equilibrium between the organic molecules and the catalyst surface. Decreases in the concentrates of dyes were analyzed by a UV-vis spectrometer (UV759, Shanghai Precision \& Scientific Instrument Co., Ltd., China). At given intervals of illumination, the samples of the reaction solution were taken out and analyzed.

\section{Results and discussion}

The powder XRD patterns of the ZnTNPc, MWCNTs, and ZnTNPc-MWCNTs composites are shown in Fig. 2. Compared with pure ZnTNPc, the diffraction peaks of the ZnTNPc-MWCNTs were broad and weak, indicating a small crystal size or poor crystallinity of ZnTNPc in the ZnTNPc-MWCNTs hybrid materials. Notably, compared to pure ZnTNPc, the characteristic peaks of the ZnTNPc-MWCNTs became weak because the ZnTNPc particles were well dispersed on the surface of MWCNTs. Moreover, all of the diffraction peaks of ZnTNPcMWCNTs could be indexed as ZnTNPc. Therefore, the XRD patterns indicated that the hybrid catalysts of ZnTNPc-MWCNTs were prepared successfully.

Figure 3 shows the SEM images of oxidized-MWCNTs and ZnTNPc-MWCNTs hybrid materials. The SEM image of ZnTNPc-MWCNTs hybrid materials showed an appreciable increase in thickness (Fig. 2b), confirming the formation of nanohybrids. It can be seen clearly that ZnTNPc particles are well dispersed in each threadlike MWCNTs matrix (Fig. 3b), showing good miscibility with MWCNTs.

The TEM morphological features of the oxidizedMWCNTs, ZnTNPc-MWCNTs and pure ZnTNPc are 


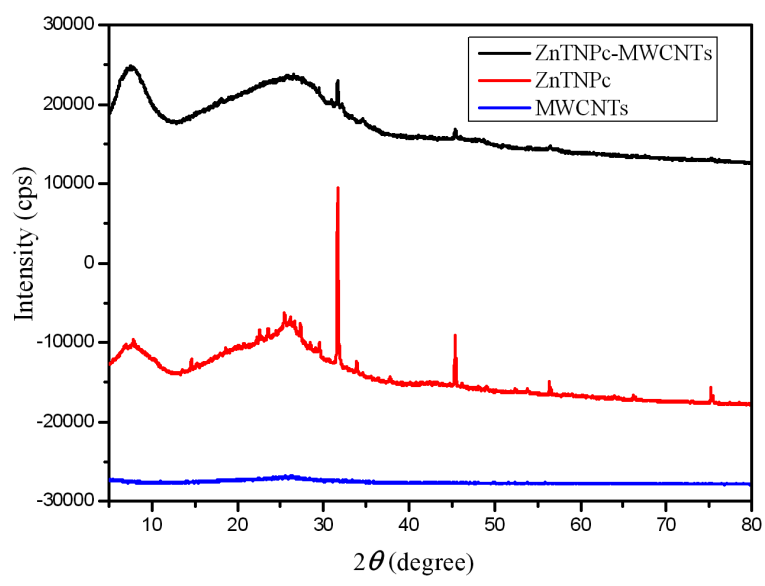

Fig. 2. XRD patterns of oxidized MWCNTs, ZnTNPc-MWCNTs, and ZnTNPc.

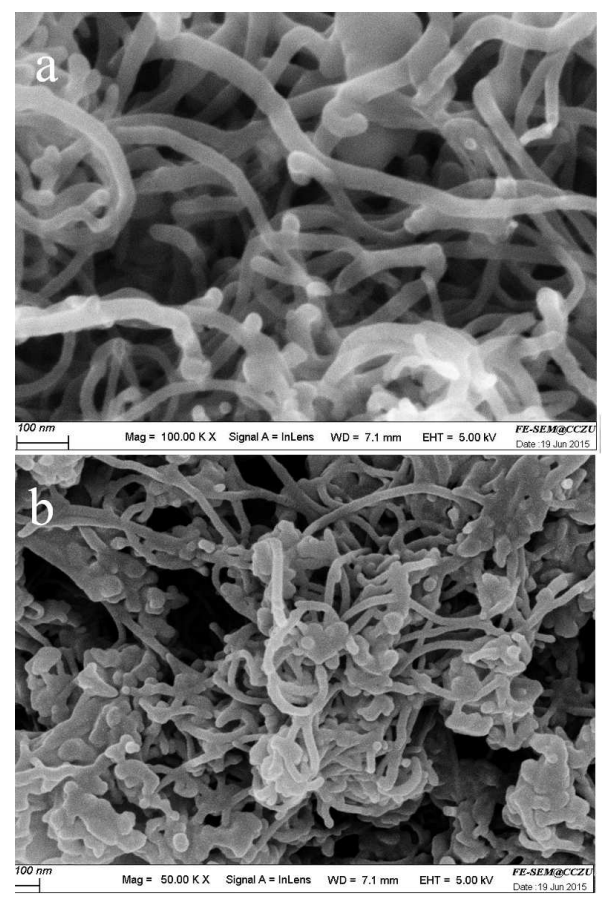

Fig. 3. SEM images of MWCNTs (a), ZnTNPc/MWCNTs composites (b).

shown in Fig. 4. It was found that each oxidizedMWCNTs was mainly long and folded pipe and its diameter was in the range of $20-30 \mathrm{~nm}$ (Fig. 4a). The resulting ZnTNPc-MWCNTs hybrid materials exhibited a stretched and shortened feature with phthalocyanines uniformly assembling on the convex surface of MWCNTs (Fig. 4b). However, pure ZnTNPc exhibited poor dispersibility with clusters congregated while the density of ZnTNPc on the MWCNTs was improved (Fig. 4c).

The ZnTNPc-MWCNTs can be further confirmed by UV-vis diffuse reflectance spectra. As shown in Fig. 5, the UV-vis diffuse reflectance spectra of ZnTNPc showed a good absorption on the wavelength of light about 468 and $628 \mathrm{~nm}$. The ZnTNPc-MWCNTs composites showed

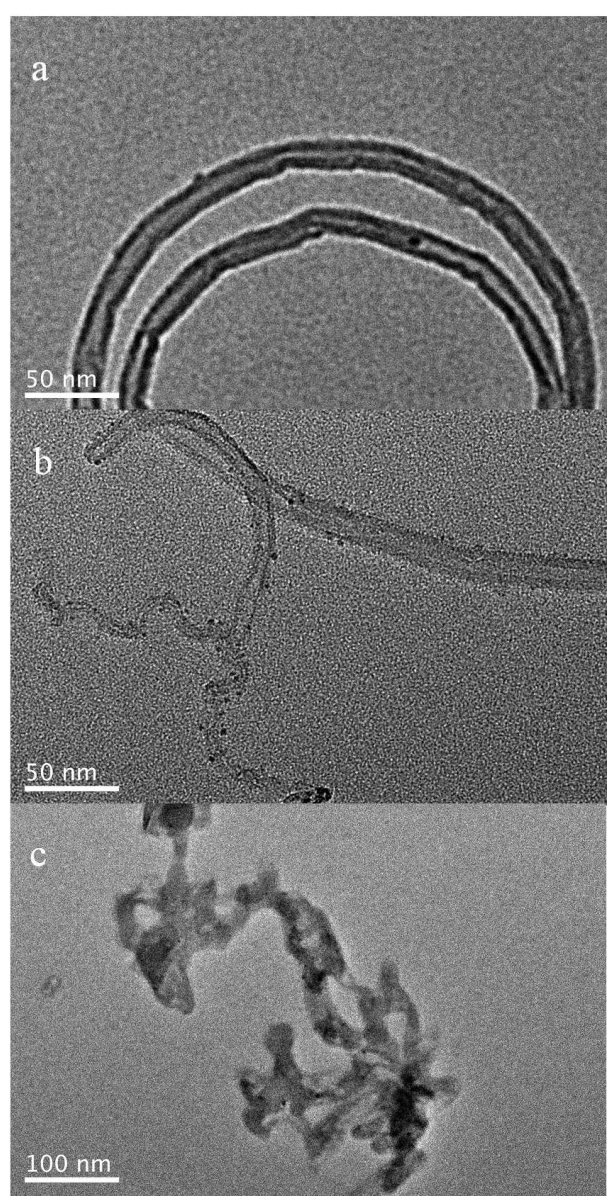

Fig. 4. TEM images of oxidized-MWCNTs (a), ZnTNPc-MWCNTs (b) and pure ZnTNPc (c).

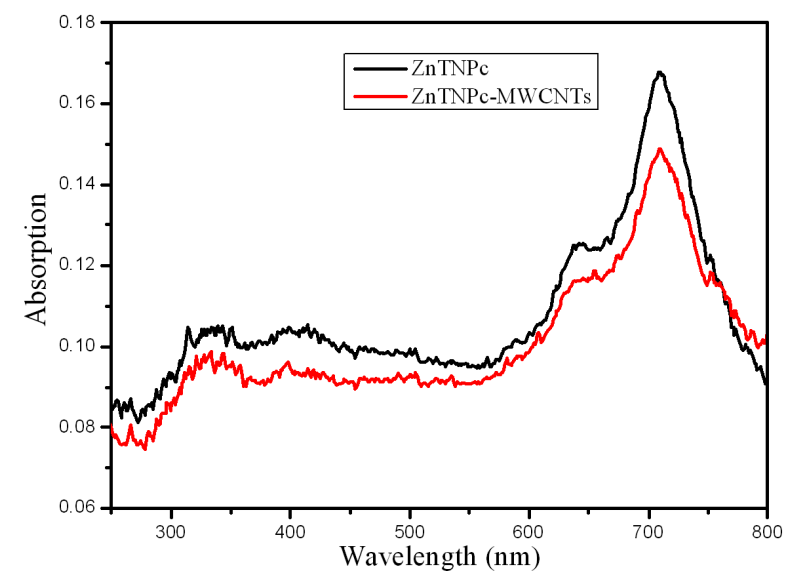

Fig. 5. UV-vis diffuse reflectance spectra of ZnTNPc and ZnTNPc-MWCNTs.

much broader and weaker absorption band through the entire visible-light region, which may be attributed to the deaggregation of ZnTNPc immobilized on the surface of MWCNTs.

The FTIR spectra of oxidized-MWCNTs, ZnTNPc, and ZnTNPc-MWCNTs are shown in Fig. 6. Carboxylic acid-functionalized MWCNTs showed two strong absorp- 


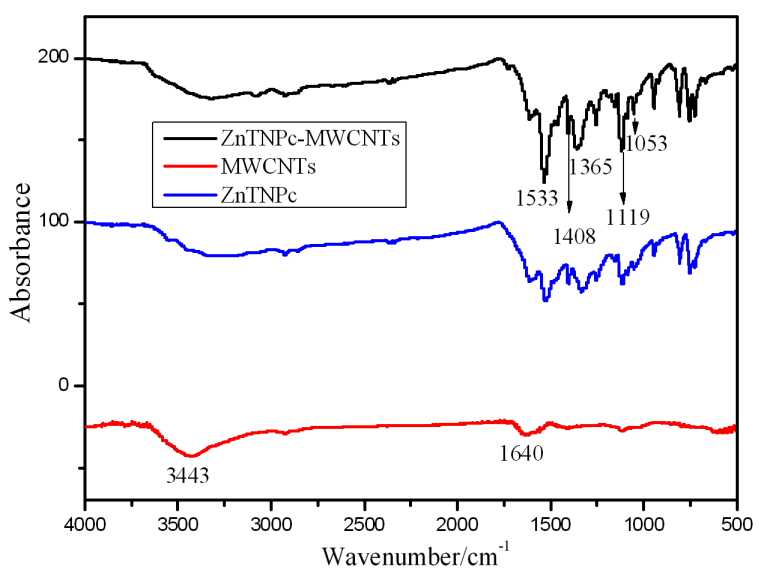

Fig. 6. FTIR spectrum of oxidized-MWCNTs , ZnTNPc and ZnTNPc-MWCNTs.

tion bands at 3443 and $1640 \mathrm{~cm}^{-1}$, associating with $\mathrm{O}-\mathrm{H}$ stretching and $\mathrm{C}=\mathrm{O}$ stretching in carboxyl, respectively. For ZnTNPc, the peaks at 1408, 1119, $1053 \mathrm{~cm}^{-1}$ are assignable to the skeleton stretching of ZnTNPc. ZnTNPc peaks appear at $1533 \mathrm{~cm}^{-1}$ and $1365 \mathrm{~cm}^{-1}(\mathrm{~N}=\mathrm{O}$ stretching vibrations of benzene). These peaks appear in ZnTNPc-MWCNTs but cannot be found in oxidizedMWCNTs, confirming the anchoring of ZnTNPc complex on the MWCNTs solid matrix.

The thermal stability of ZnTNPc-MWCNTs was further estimated by thermogravimetric analysis (Fig. 7). The ZnTNPc shows a characteristic thermogram with the first weight loss occurring between $300^{\circ} \mathrm{C}$ and $330^{\circ} \mathrm{C}$, and the second weight loss occurs from $330^{\circ} \mathrm{C}$ to $480^{\circ} \mathrm{C}$. Based on the weight loss and residue quantity at $600^{\circ} \mathrm{C}$ the weight percentage of $\mathrm{ZnTNPc}-\mathrm{MWCNT}$ s hybrid materials and the pure ZnTNPc is about $46.2 \%$ and $15.2 \%$, respectively. Thus, the results indicated that $\mathrm{ZnPc}-$ MWCNTs possessed good thermal stability under $600^{\circ} \mathrm{C}$.

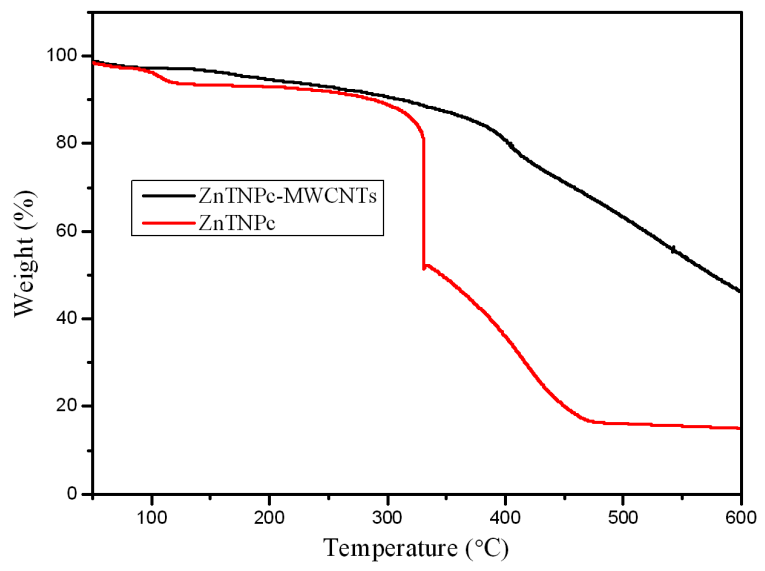

Fig. 7. TGA curves of ZnTNPc-MWCNTs, pure ZnT$\mathrm{NPc}$ recorded at a rate of $20^{\circ} \mathrm{C} / \mathrm{min}$.

To test the photocatalytic activity of ZnTNPcMWCNTs hybrid materials for the degradation of organic pollutions, RB was selected as a representative dye pol- lutant of industrial waste waters. The photocatalytic activities of the ZnTNPc-MWCNTs hybrid materials were evaluated by the degradation of RB in aqueous solution under visible-light irradiation. Before studying and comparing the activities of the ZnTNPc-MWCNTs hybrid materials, the bleaching of RB in the absence of any catalysts was first examined. As shown in Fig. 8, RB degradation without any photocatalyst was performed, and the results illustrated that the degradation of $\mathrm{RB}$ was very slow in the absence of photocatalyst under visible-light irradiation, only $1 \%$ of RB was degraded under the irradiation with visible light. Next, the photocatalytic activities of MWCNTs were investigated. It can be clearly seen that the MWCNTs had almost no photocatalytic activity under the visible-light irradiation, except decent adsorption for RB (13.6\%), which could attributed to the high specific surface area of the carbon nanotubes. Irradiation of aqueous solutions of RB $(25 \mathrm{mg} / \mathrm{L})$ after the ZnTNPc immobilized onto MWCNTs led to an obvious decrease of $\mathrm{RB}$ concentration. The excellent photoactivity depended on the structure of the immobilized phthalocyanines, implying that the degradation of RB was initiated by its reaction with the reactive oxygen species produced upon exciting the phthalocyanines with the appropriate wavelength of light. On the other hand, pure ZnTNPc barely showed activity for RB photodegradation (53.7\%) due to the aggregation of ZnTNPc decreased the photoactivity, while ZnTNPc-MWCNTs demonstrates good photocatalytic activity with a degradation rate of $0.3492 \mathrm{~h}^{-1}$.

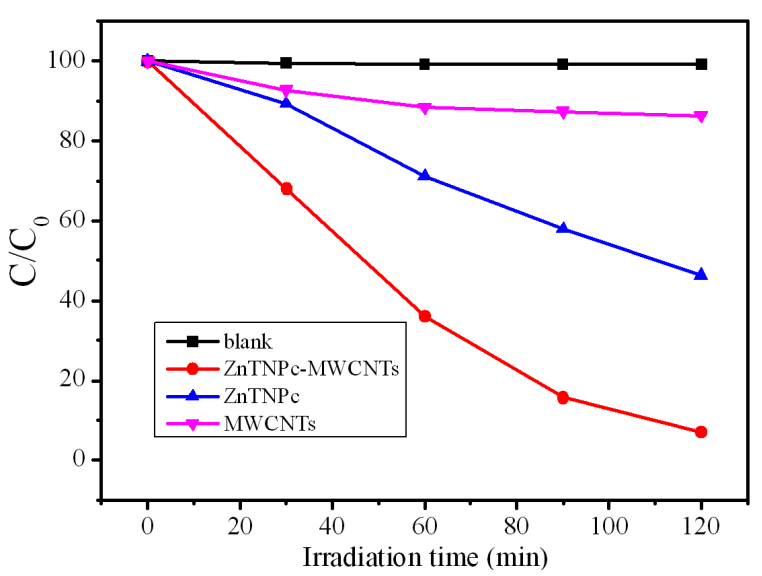

Fig. 8. Photocatalysis of RB by different photocatalysts with the same weight under visible-light irradiation.

The linearity of plots of $\ln \left(C_{0} / C_{t}\right)$ against time (from the kinetic regime) (Fig. 9) suggested that the degradation of RB using the pure ZnTNPc or ZnTNPcMWCNTs followed the pseudo-first order kinetics, here $C_{0}$ is the equilibrium concentration of $\mathrm{RB}$ after $30 \mathrm{~min}$ dark adsorption, $C_{t}$ is the $\mathrm{RB}$ concentration remaining in the solution at irradiation time ( $\min )$, and $k$ is the observed rate constant. The introduction of MWCNTs promoted the degradation of RB effectively. The $k$ value was $0.3492 \mathrm{~h}^{-1}$, which was 3.3 and 149 times 


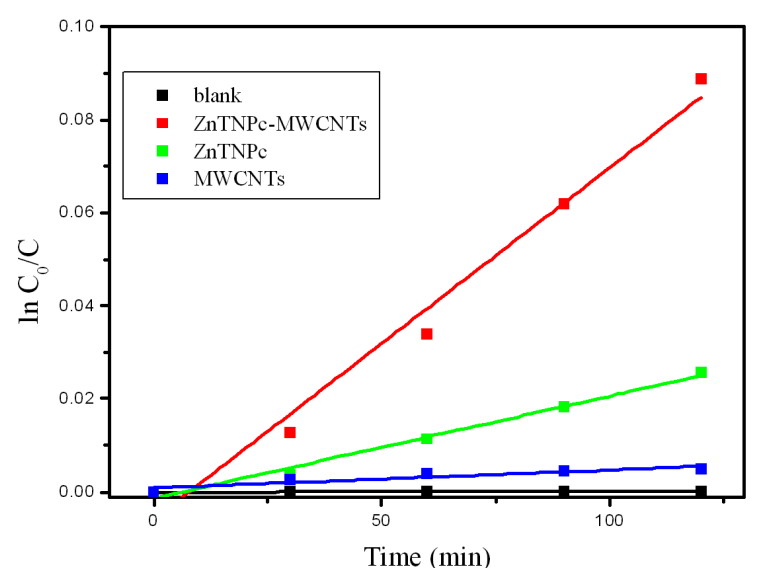

Fig. 9. Kinetic plot for ZnTNPc-MWCNTs, ZnTNPc, MWCNTs, and blank in photooxidation of RB.

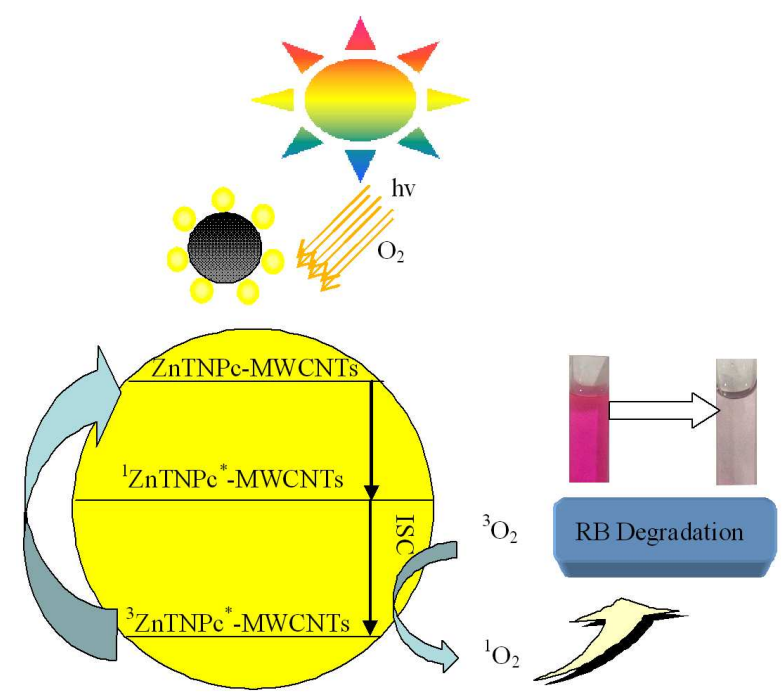

Fig. 10. Proposed mechanism of RB photodegradation in ZnTNPc-MWCNTs under visible-light irradiation.

higher than that of pure ZnTNPc $\left(0.081 \mathrm{~h}^{-1}\right)$ and MWCNTs $\left(0.00232 \mathrm{~h}^{-1}\right)$, respectively. Obviously, the coupling of ZnTNPc and MWCNTs by the method of ultrasonic impregnation created an excellent hybrid photocatalyst. The enhanced photoactivity may be ascribed to the improvement in charge transmission between the MWCNTs and $\mathrm{ZnTNPc}$ prolonging the lifetime of charge carriers. Moreover, the photoactivity of the hybrid photocatalyst was also affected by the surface contact between particles. The ZnTNPc-MWCNTs hybrid materials had higher contact interfaces between ZnTNPc and MWCNTs, resulting in higher photocatalytic efficiency.

Table I shows the photocatalytic activity of asprepared of ZnTNPc-MWCNTs hybrid photocatalyst by comparison of the metallophthalocyanine and other metal photocatalysts. It was found that $93 \%$ of the $\mathrm{RB}$ was degraded under the visible-light irradiation with ZnTNPc-MWCNTs as photocatalyst, confirming that the as-prepared ZnTNPc-MWCNTs hybrid materials
TABLE I

Photocatalytic activities toward the degradation of RB of some reported catalysts. A - reaction time [min], $\mathrm{B}$ - removal percentage [\%], $\mathrm{C}$ - concentration $[\mathrm{g} / \mathrm{L}]$.

\begin{tabular}{c|c|c|c|c}
\hline \hline Photocatalyst & A & B & C & Ref. \\
\hline $\mathrm{CuTNPc} / \mathrm{TiO}_{2}$ & 240 & 87 & 10 & 17 \\
$\mathrm{FeTNPc}$ & 300 & 90 & 10 & 27 \\
$\mathrm{TiO}_{2} / \mathrm{RGO}$ & 210 & 75 & 10 & 28 \\
$\mathrm{NiO}$ & 180 & 80 & 10 & 29 \\
$\mathrm{CdPc} / \mathrm{PAN}$ & 420 & 85 & 10 & 30 \\
$\mathrm{Bi}_{5} \mathrm{O}_{7} \mathrm{Br}$ & 120 & 85 & 11 & 31 \\
ZnTNPc-MWCNTs & 120 & 93 & 25 & this work
\end{tabular}

showed good photocatalytic performance.

$$
\begin{aligned}
& \mathrm{ZnTNPc}-\mathrm{MWCNTs} \stackrel{h v}{\longrightarrow} \\
& { }^{1} \mathrm{ZnTNPc}^{*}-\mathrm{MWCNT}, \\
& { }^{1} \mathrm{ZnTNPc}^{*}-\mathrm{MWCNTs} \stackrel{\mathrm{ISC}}{ } \\
& { }^{3} \mathrm{ZnTNPc}^{*}-\mathrm{MWCNTs}, \\
& { }^{3} \mathrm{O}_{2}+{ }^{3} \mathrm{ZnTNPc}^{*}-\mathrm{MWCNTs} \longrightarrow \\
& \mathrm{ZnTNPc}-\mathrm{MWCNTs}+{ }^{1} \mathrm{O}_{2}, \\
& { }^{1} \mathrm{O}_{2}+\mathrm{RB} \longrightarrow \text { oxidation product. }
\end{aligned}
$$

In heterogeneous reaction for ZnTNPc-MWCNTs hybrid materials under visible-light irradiation, the generation of singlet oxygen is an adopted photocatalytic mechanism [17]. On the basis of the above results and the earlier reports on the photocatalytic oxidation of pollutants, a proposed mechanism of visible lightinduced photodegradation of $\mathrm{RB}$ with the $\mathrm{ZnTNPc}^{-}$ MWCNTs hybrid materials is elucidated in Fig. 10. The ZnTNPc-MWCNTs was excited by visible-light irradiation to form the single line excitation state $\left({ }^{1} \mathrm{ZnTNPc}^{*}\right.$ MWCNTs) (Eq. (1)), and then ${ }^{1} \mathrm{ZnTNPc}^{*}-\mathrm{MWCNTs}$ (Eq. (2)) can turn into the excited triplet states sensitizer of ${ }^{3} \mathrm{ZnTNPc}^{*}-\mathrm{MWCNTs}$ by irradiation and intersystem crossing (ISC). ${ }^{3} \mathrm{ZnTNPc}^{*}-\mathrm{MWCNTs}$ can interact with the ground state triplet oxygen $\left({ }^{3} \mathrm{O}_{2}\right)$ (Eq. (3)) to generate the highly active singlet oxygen $\left({ }^{1} \mathrm{O}_{2}\right)$, resulting in the decomposition of RB (Eq. (4)).

\section{Conclusions}

In summary, novel ZnTNPc-MWCNTs hybrid materials were facilely prepared through a simple and effective ultrasonic impregnation route. Furthermore, the ZnTNPc-MWCNTs exhibited excellent photocatalytic activities for RB under visible-light irradiation. The asprepared ZnTNPc-MWCNTs powders displayed much higher visible-light photocatalytic activity than pure ZnTNPc or MWCNTs under visible light irradiation. A possible photocatalytic mechanism for "singlet oxygen process" was suggested. Hence, ZnTNPc-MWCNTs can be considered as potent catalyst that can be utilized for the waste water treatment process in real time applications. 


\section{Acknowledgments}

This work was financially supported by Natural Science Foundation of Jiangsu Province, China (No. BK20150259) and Natural Science Foundation of Changzhou City (CJ20140053).

\section{References}

[1] Y. Ding, F. Yang, L. Zhu, N. Wang, H. Tang, Appl. Catal. B Environ. 164, 151 (2015).

[2] G. Melinte, M. Baia, D. Georgescu, L. Baia, V. Iancu, L. Diamandescu, T. Popescu, L.C. Cotet, L. BarbuTudoran, V. Danciu, S. Simon, Acta Phys. Pol. A 121, 208 (2012).

[3] G.H. Chen, Y. Wang, J.H. Zhang, C.L. Wu, H.D. Liang, H. Yang, J. Nanosci. Nanotechnol. 12 3799 (2012)

[4] R. Azimirad, S. Safa, O. Akhavan, Acta Phys. Pol. A 127, 1727 (2015).

[5] A. Fujishima, Nature 238, 37 (1972).

[6] M. Tuncer, B. Ozdemir, Acta Phys. Pol. A 125, 608 (2014).

[7] H. Fang, Y. Gao, G. Li, J. An, P.K. Wong, H. Fu, S. Yao, X. Nie, T. An, Environ. Sci. Technol. 47, 2704 (2013)

[8] P. Li, Z. Wei, T. Wu, Q. Peng, Y. Li, J. Am. Chem. Soc. 133, 5660 (2011).

[9] P. Raizada, P. Singh, A. Kumar, G. Sharma, B. Pare, S.B. Jonnalagadda, P. Thakur, Appl. Catal. A Gen. 486, 159 (2014).

[10] Z. Yu, B. Yin, F. Qu, X. Wu, Chem. Eng. J. 258, 203 (2014).

[11] X. Dai, M. Xie, S. Meng, X. Fu, S. Chen, Appl. Catal. B Environ. 158-159, 382 (2014).

[12] C. Chung, Y.K. Kim, D. Shin, S.R. Ryoo, B.H. Hong, D.H. Min, Acc. Chem. Res. 46, 2211 (2013).

[13] G. Qin, J. Zhang, C. Wang, J. Alloys Comp. 635 , 158 (2015).

[14] A. Solhy, B.F. Machado, J. Beausoleil, Y. Kihn, F. Goncalves, M.F.R. Pereira, J.J.M. Orfao, J.J. Figueiredo, J.L. Faria, P. Serp, Carbon 46, 1194 (2008).
[15] S. Wang, X. Shi, G. Shao, X. Duan, H. Yang, T. Wang, J. Phys. Chem. Solids 69, 2396 (2008).

[16] H. Vu, F. Goncalves, R. Philippe, E. Lamouroux, M. Corrias, Y. Kihn, D. Plee, P. Kalck, P. Serp, J. Catal. 240, 18 (2006).

[17] M.Y. Zhang, C.L. Shao, Z.C. Guo, Z.Y. Zhang, J.B. Mu, P. Zhang, T.P. Cao, Appl. Mater. Interfaces 3, 2573 (2011).

[18] E. Marais, R. Klein, E. Antunes, T.J. Nyokong, J. Mol. Catal. A Chem. 261, 36 (2007).

[19] G. Palmisano, M.C. Gutierrez, M.L. Ferrer, M.D. GilLuna, V. Augugliaro, S. Yurdakal, M. Pagliaro, J. Phys. Chem. C 112, 2667 (2008).

[20] H.J. Mackintosh, P.M. Budd, N.B. McKeown, J. Mater. Chem. 18, 573 (2008).

[21] S. Makhseed, F. Al-Kharafi, J. Samuel, B. Ateya, Catal. Commun. 10, 1284 (2009).

[22] Z. Huang, B. Zheng, S. Zhu, Y. Yao, Y. Ye, W. Lu, W. Chen, Mater. Sci. Semicond. Process. 25, 148 (2014).

[23] Y. Yao, Z. Huang, B. Zheng, S. Zhu, W. Lu, W. Chen H. Chen, Curr. Appl. Phys. 13, 1738 (2013).

[24] W. Lu, C. Sun, Q. Lu, N. Li, D. Wu, Y. Yao, W. Chen, Sci. China Chem. 55, 1108 (2012).

[25] C. Zhou, Y. Liu, X. Zhao, Inorg. Chim. Acta 425, 11 (2015).

[26] M. Gao, N. Li, W. Lu, W. Chen, Appl. Catal. B Environ. 147, 805 (2014).

[27] Z.C. Guo, J.B. Mu, B. Chen, Ceram. Int. 41, 4916 (2015).

[28] L.X. Zhang, J. Zhang, H.F. Jiu, C.H. Ni, X. Zhang, M.L. Xu, J. Phys. Chem. Solids 86, 82 (2015).

[29] M. Fereshteh, R.M. Mohammad, S. Faezeh, S.N. Masoud, RSC Adv. 4, 27654 (2014).

[30] Z.C. Guo, C.L. Shao, J.B. Mu, M.Y. Zhang, Z.Y. Zhang, Z. Peng, B. Chen, Y.C. Liu, Catal. Commun. 12, 880 (2011).

[31] Y.R. Su, C.H. Ding, Y.L. Yuan, H. Wang, L.Q. Ye, X.L. Jin, H.Q. Xie, C. Liu, Appl. Surf. Sci. 346, 311 (2015). 